\title{
A Proposed Mechanism for the Solid-state Polymerization of Cyclic Monomers in Lattice Defects
}

\author{
Geoffrey Charles EASTMOND* \\ Department of Polymer Chemistry, Faculty of Engineering, Kyoto University, \\ Kyoto, Japan.
}

(Received July 3, 1972)

\begin{abstract}
The crystal structures of trioxane, tetroxane and pentoxane have been examined with a view to identifying the most probable edge dislocations in their crystals. The results of this examination have been correlated with observed directions of polymer growth in the crystalline monomers. From the results it is suggested that initiation of solid-state polymerization may occur in edge dislocations rather than in the perfect monomer lattice.
\end{abstract}

KEY WORDS Defect / Dislocation / Pentoxane / Polymerization

/ Solid State / Tetroxane / Trioxane /

\section{INTRODUCTION}

About 1960 Okamura, et al., discovered that radiation-induced solid-state polymerizations of single crystals of trioxane ${ }^{1}$ and other cyclic monomers $^{2}$ yielded crystalline polymers. Subsequently it was observed that crystalline poly(oxymethylene) produced by solid-state polymerization of trioxane has its $c$-axis orientated parallel to the $c$-axis of the parent monomer crystal, ${ }^{3}$ and similar observations have been reported for other cyclic monomers. These observations lent strong support to ideas that solid-state polymerizations proceed through perfect monomer crystal lattices and that monomer lattice structures are of paramount importance in controlling the polymerizations. It was visualised that growth of crystalline polymer proceeded by reaction of successive monomer molecules in certain preferred directions and that correct orientation of monomer molecules was necessary for polymerization. Kinetic data were also interpreted on this basis. ${ }^{4}$

The above situation contrasts markedly with that appertaining to the solid-state polymerization of vinyl monomers, which almost invariably lead to the formation of amorphous polymers. Various studies have demonstrated that lattice defects, particularly dislocations, are the

* Permanent address, to which correspondence should be addressed: Donnan Laboratories, The University of Liverpool, Liverpool, England. preferred reaction sites for polymerization of such monomers. ${ }^{5}$ In addition, sufficient molecular mobility is an important requirement for reaction. ${ }^{6}$

Many researchers now take the view that two types of solid-state polymerization exist; (i) reactions leading to amorphous polymer in which reaction occurs in lattice defects, and (ii) reactions leading to crystalline polymer where reaction involves direct lattice control, including the polymerizations of trioxane, etc. It is the purpose of this communication to demonstrate that the solid-state polymerizations of trioxane and related monomers can be satisfactorily explained in terms of initiation in lattice defects (especially edge dislocations), the structures of which are controlled by the monomer lattice structure, so that there may be no sharp distinction between the two types of polymerization.

\section{DIFFICULTIES ASSOCIATED WITH THE LATTICE CONTROL THEORY}

Since the original studies referred to above, detailed studies of solid-state polymerizations of trioxane and other cyclic oligomers of formaldehyde have brought to light certain difficulties associated with the simple mechanism of lattice control.

Solid-state polymerizations of trioxan, tetroxane and pentoxane only occur rapidly at tem- 
peratures near to the melting points of the respective monomers ${ }^{7}$. Even at room temperature trioxane is a soft crystal and at higher temperatures, i.e., about $50^{\circ} \mathrm{C}$, where polymerization is rapid, molecular motions are very extensive ${ }^{8}$. Similarly, extensive molecular motions are present in tetroxane at polymerization temperatures ${ }^{7}$. While high molecular mobility is apparently essential for effective and rapid polymerization of these monomers, and may assist molecular reorientations during polymerization, it is difficult to reconcile such high molecular mobility with lattice-controlled propagation. It has also been demonstrated that trioxane will polymerize in the melt ${ }^{9}$.

Apart from the above considerations the crystallographic evidence itself poses certain problems, the first of these is associated with polymer growth. Polymerization in single crystals of trioxane, tetroxane and pentoxane all produce crystalline poly(oxymethylene), in the form of a $29 / 16$ helix $^{10}$ or virtually identical $9 / 5$ helix $^{11,12}$, in a preferred direction in the monomer crystal. However, in all cases the polymer structures appear 'twinned,' with sub-crystals of polymer growing in other specific directions through the monomer lattices ${ }^{10-12}$. Thus, formation of crystalline polymer is not restricted to growth through monomer molecules in one specific arrangement.

The second, and more serious difficulty, arises from the lack of correspondence between the lattice structures of monomer and polymer. Chatani, et al., ${ }^{11,12}$ have summarised the intermolecular distances in crystals of trioxane, tetroxane and pentoxane. While formation of crystalline poly(oxymethylene) is normally accompanied by a contraction perpendicular to the $c$-axis of the polymer helix, a considerable expansion along the axis of the helix is required. For example, in pentoxane the distance between the centres of the molecules in the main growth direction is only $6.74 \AA$ while five $\left(\mathrm{CH}_{2}-\mathrm{O}\right)$ units in the polymer helix occupy $9.65 \AA$. Thus a polymer chain cannot grow through the lattice without causing considerable disruption of that lattice at the addition of each monomer molecule.

The difficulties outlined above have been re- cognised by workers in the field who have proposed modifications to the simple mechanism in attempts to overcome them. It has been proposed that formation of sub-crystals of polymer can help to relieve strain introduced by polymerization in the major growth direction ${ }^{11}$. However, in pentoxane sub-crystal formation does not occur in a direction likely to relieve stress ${ }^{12}$ but in an even more congested direction where the distance between the centres of monomer molecules is only $5.62 \AA$. Experimentally it has been found that the extent of sub-crystal formation decreases as the polymerization temperature increases, and it has been suggested that at the higer temperatures the strain responsible for sub-crystal formation is relieved by large-scale molecular motions or diffusion ${ }^{7,11}$. Other modifications to the simple mechanism, based on geometrical considerations and on high molecular mobility and vapour pressure of the monomers, have been considered ${ }^{7,11-14}$

In contrast to the crystallographic studies described above, all of which used single crystals of the monomers, Adler carried out experiments using polycrystalline samples of trioxan $e^{15}$. These experiments showed that crystalline polymer fibrils grew across intercrystalline boundaries without change in direction, even though the monomer crystals were in different orientations, demonstrating that growth of crystalline polymer does not require specific orientation of monomer.

From the results of numerous studies on single crystals there can be no doubt that in single monomer crystals crystalline polymer only forms in certain specific orientations. On the other hand, Adler's data indicates that propagation is not controlled by the monomer lattice. The two sets of data can be reconciled by assuming that the polymerization is initiated in certain specific directions and that propagation then occurs by addition of monomer to polymer already in the form of a helix, resulting in the continued growth of the polymer in the initial direction. This interpretation implies that initiation occurs at specific sites within the monomer crystals ${ }^{15}$, presumably imperfections. This conclusion is consistent with the observation of Bassett ${ }^{16}$ that $\{001\}$ sub-grain boundaries 


\section{G. C. EAstmond}

are preferred sites for initiation. The problem, therefore, which remains to be answered is that if propagation is not lattice controlled what factors are responsible for initiation occuring in a limited number of specific directions?

\section{A PROPOSED DEFECT MECHANISM}

From studies of ultraviolet-initiated polymerizations of vinyl monomers, we have proposed that radical formation ${ }^{17}$ and polymerization occur in dislocations ${ }^{18}$. Similar results and conclusions have been obtained by other workers on related systems ${ }^{5}$. It seems of interest to extend these ideas to polymerizations of cyclic monomers in an attempt to overcome the difficulties described in the preceding section. Dislocations are energetically favourable sites for reaction in any crystal and edge dislocations are often considered to be the most probable sites of reaction.

Dislocation theory has long been established in metal crystals and it is now becoming in-
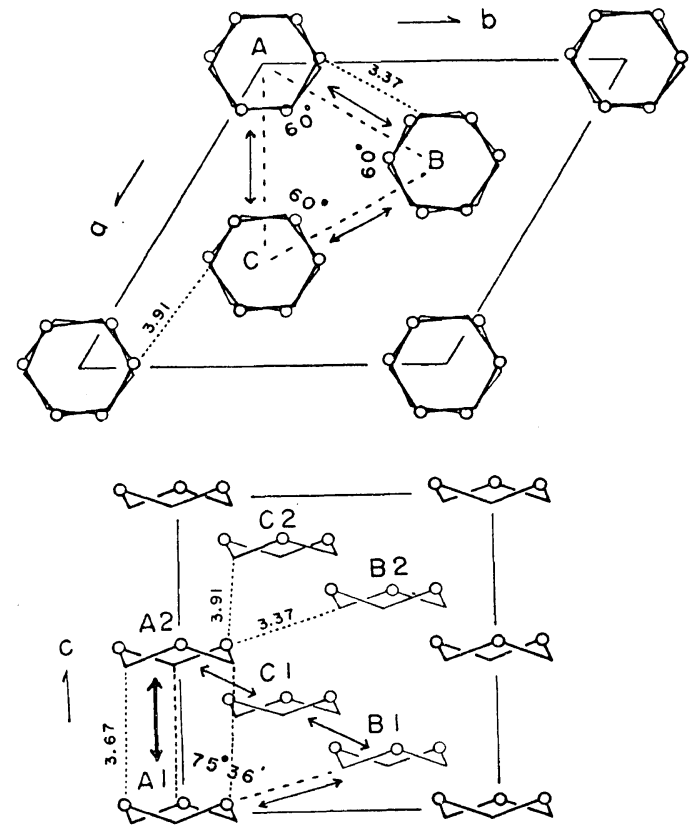

$b \sin \gamma$

Figure 1. Crystal structure of trioxane (reproduced from ref 12 by kind permission of Marcel Dekker Inc.). Thick and thin double-headed arrows indicate the main and subsidiary directions of polymer growth. creasingly apparent that dislocations also exist in organic crystals and that they have similar properties to the corresponding defects in metals. Restricting our discussion to edge dislocations, in the vicinity of the dislocation core there is a region of lattice distortion and running parallel to the dislocation line there is a void which provides free volume and probably facilitates chemical reaction. The natural location of an edge dislocation is along the intersection of a slip-plane and a plane suitable for the insertion of, or removal of, a half-plane of atoms or molecules; the dislocation line runs across the slipplane and along the terminating edge of the extra, or missing, half-plane ${ }^{19}$. No experimental study aimed at identifying dislocations in crystals of the cyclic monomers has yet been carried out, but it is possible to predict probable dislocation lines on the basis of known crystal structures. We have constructed space-filling models of crystalline trioxane, tetroxane and pentoxane. In this section we report the results of an attempt to identify the most probable dislocation lines using the models and to correlate the disloca-
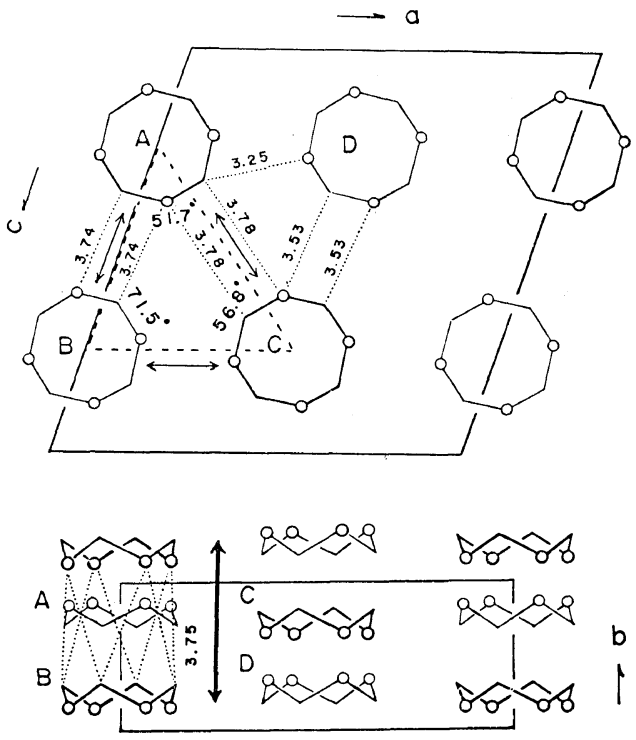

$\rightarrow a \sin \beta$

Figure 2. Crystal structure of tetroxane (reproduced from ref 12 by kind permission of Marcel Dekker Inc.). Thick and thin double-headed arrows indicate the main and subsidiary directions of polymer growth. 
tions with known preferred directions of polymer growth in single monomer crystals.

To aid discussion it is convenient to refer to the crystal structures of trioxane, tetroxane and pentoxane as presented by Chatani, et al ${ }^{11,12}$ (Figures 1, 2, and 3, respectively), in which the main and subsidiary directions of polymer growth are indicated by thick and thin doubleheaded arrows, respectively. In attempting to predict dislocations it must be remembered that crystal structure diagrams showing atomic centres misrepresent the amount of free space in the crystal; the molecules in real crystals are efficiently packed and effectively fill all available space.

In trioxane the main polymer growth direction is [001], and parallel to this growth direction are six potential slip-planes: (110), (210), $(1 \overline{2} 0),(100),(010)$ and (110). Each of these planes is associated with five planes suitable for
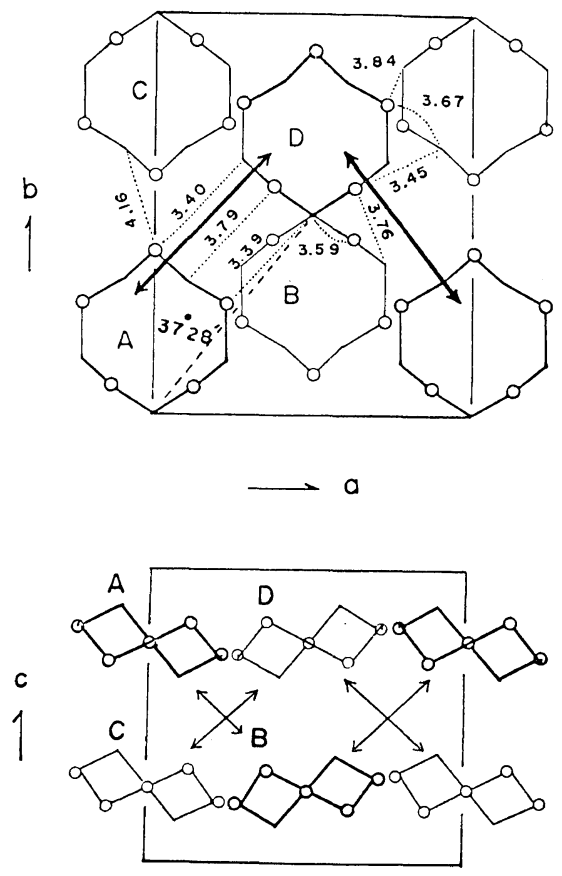

Figure 3. Crystal structure of pentoxane (reproduced from ref 12 by kind permission of Marcel Dekker Inc.). Thick and thin double-headed arrows indicate the main and subsidiary directions of polymer growth.
Table I. Planes, associated with various slipplanes, in crystalline trioxane suitable for the generation of edge-dislacation lines parallel to [001]

\begin{tabular}{rllllll}
\hline Slip-plane & $(110)$ & $(2 \overline{1} 0)$ & $(1 \overline{2} 0)$ & $(100)$ & $(010)$ & $(1 \overline{1} 0)$ \\
\hline & $(1 \overline{1} 0)$ & $(010)$ & $(100)$ & $(1 \overline{2} 0)$ & $(2 \overline{1} 0)$ & $(110)$ \\
& $(2 \overline{1} 0)$ & $(1 \overline{2} 0)$ & $(2 \overline{1} 0)$ & $(1 \overline{1} 0)$ & $(1 \overline{1} 0)$ & $(100)$ \\
& $(1 \overline{2} 0)$ & $(110)$ & $(110)$ & $(010)$ & $(100)$ & $(010)$ \\
& $(010)$ & $(1 \overline{1} 0)$ & $(1 \overline{1} 0)$ & $(2 \overline{1} 0)$ & $(1 \overline{2} 0)$ & $(1 \overline{2} 0)$ \\
& $(100)$ & $(100)$ & $(010)$ & $(110)$ & $(110)$ & $(2 \overline{1} 0)$ \\
\hline
\end{tabular}

the introduction or removal of half-planes of molecules, thus generating edge dislocations with the dislocation lines running parallel to the [001] direction. The planes associated with each slipplane are given in Table $I$, in each case the first plane mentioned makes an angle of $90^{\circ}$ with the slip-plane, the next two angles of $60^{\circ}$ and the final two angles of $30^{\circ}$ with the slipplane. Of the potential slip-planes mentioned the (110), $(2 \overline{1} 0)$ and $(1 \overline{2} 0)$ are probably the major slip-planes. The Burger's vectors in (100), $(010)$ and $(1 \overline{1} 0)$ are long and there is considerable overlap of molecules in these planes which will hinder slip, as shown in Fig. 4.

Subsidiary polymer growth directions in tri-

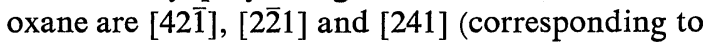
propagation in the directions $\mathrm{A} \leftrightarrow \mathrm{C}, \mathrm{B} \leftrightarrow \mathrm{C}, \mathrm{A} \leftrightarrow$ $B$ in Figure 1). Taking the [421] direction as an example, four slip-planes possibly suitable for generating edge dislocations require consideration, each of which is associated with planes suitable for insertion, or removal, of half-planes of molecules. Of the four possible slip-planes, detailed in Table II, the (012), (1112) and (1120) are perhaps the most suitable. The (104) plane is probably the least suitable slip-plane for

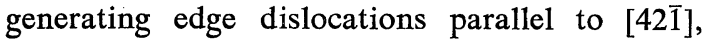
since it is almost certainly not suitable for true slip but only pencil-glide in [421] ; it may, however be suitable as a source of half-planes.

Table II. Planes associated with various slipplanes suitable for generating edge-dislocation lines parallel to $[42 \overline{1}]$ in crystalline trioxane

\begin{tabular}{ccccc}
\hline Slip-plane & $(012)$ & $(1 \overline{1} 2)$ & $(1 \overline{2} 0)$ & $(104)$ \\
\hline & $(1 \overline{2} 0)$ & $(1 \overline{2} 0)$ & $(012)$ & $(1 \overline{2} 0)$ \\
& $(1 \overline{1} 2)$ & $(012)$ & $(1 \overline{1} 2)$ & $(012)$ \\
& $(104)$ & $(104)$ & $(104)$ & $(1 \overline{1} 2)$ \\
\hline
\end{tabular}




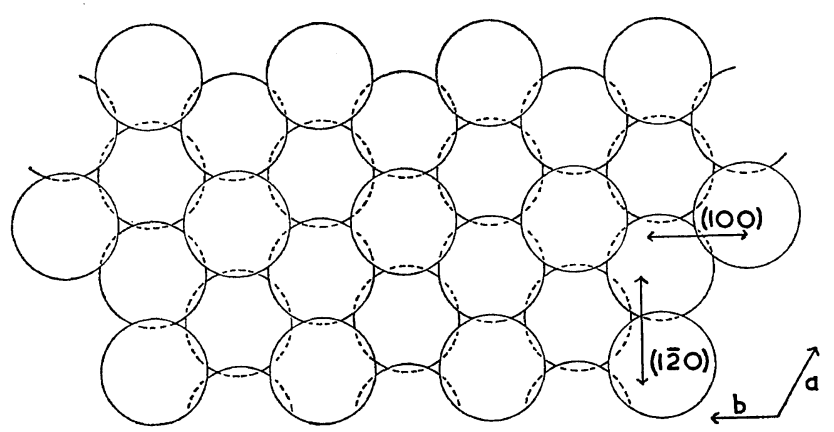

Figure 4. Schematic representation of a trioxane crystal. Slip, in the directions indicated by double-headed arrows, will be more difficult in (100) than in $(1 \overline{2} 0)$ as a result of molecular overlap.

Planes suitable for removal of half-planes to generate edge-dislocation lines running in [421] are detailed in Table II. Similarly, edge dislocations can be generated parallel to the equivalent growth directions [2 $2 \overline{1} 1]$ and [241].

Examination of Tables I and II shows that depending on how the edge of a half-plane terminates it may be used in conjunction with different slip-planes to generate dislocation lines parallel to polymer growth directions. The (120) plane can be used to generate edge-dislocation lines running in the main or subsidiary polymer growth directions.

It is necessary to consider the possible generation of edge dislocations in directions other than observed polymer growth directions. One such possibility is [210], since this lies in the (210) plane-a probable slip-plane. However, there are no other planes which can terminate in the [210] direction suitable for removal of half-planes of molecules. An apparently obvious slip-plane is (001) but this is not suitable on account of the staggered positions of the molecules at the (001) plane (Figure 5). Other possibilities which should be considered are dislocations running in [100] and [010], using the (101) and (100) planes as slip-planes and inserting half-planes in (021) and (201), respectively. These alternatives are again unlikely since (010), (100) are not good slip-planes for reasons men-

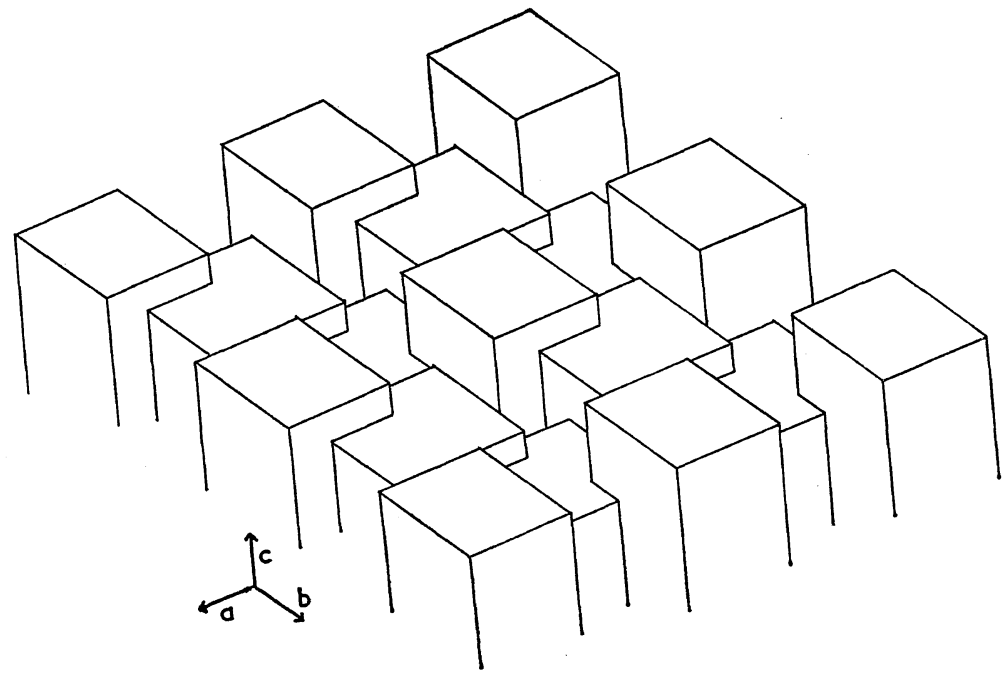

Figure 5. Schematic representation of a section of the (001) plane in trioxane, showing the unsuitability of (001) to act as a slip plane. 
Table III. Planes associated with various slipplanes in tetroxane crystals suitable for generating edge-dislocation lines parallel to [010]

\begin{tabular}{ccccc}
\hline Slip plane & $(100)$ & $(001)$ & $(10 \overline{1})$ & $(101)$ \\
\hline & $(001)$ & $(100)$ & $(101)$ & $(10 \overline{1})$ \\
$(10 \overline{1})$ & $(10 \overline{1})$ & $(001)$ & $(001)$ \\
& $(101)$ & $(101)$ & $(100)$ & $(100)$ \\
\hline
\end{tabular}

tioned earlier.

Tetroxane crystals show a similar series of possibilities. The main polymer growth direction in the monomer crystal is [010]. Edge-dislocation lines parallel to [010] can be generated by inserting partial planes of molecules in the planes given in Table III, terminating in the appropriate slip-planes. Of the possible slip-planes (101) is probably not a satisfactory slip-plane for the same reasons as the (100) plane in trioxane.

Subsidiary polymer growth directions in tetroxane are [001], [100] and [101], perpendicular to the major growth direction, and parallel to the $a$-axis, the $c$-axis and to one diagonal of the plane formed by the $a$ - and $c$-axes, respectively. These directions approximate to proceeding through the crystal in a zigzag manner (alternately up and down the $b$-axis, with no net vector along the $b$-axis) through molecules designated $B \leftrightarrow C \leftrightarrow B^{\prime} \leftrightarrow C^{\prime}, A \leftrightarrow B \leftrightarrow A^{\prime} \leftrightarrow B^{\prime}$, and $A \leftrightarrow$ $\mathrm{C} \leftrightarrow \mathrm{A}^{\prime} \leftrightarrow \mathrm{C}^{\prime}$ (Figure 2), where a dash refers to an equivalent molecule in an adjacent unit cell. Planes suitable for insertion of partial planes to give dislocation lines along the various subsidiary growth directions are given in Table IV, under the appropriate slip-planes.

(010) is probably not a satisfactory plane for slip and suggested dislocations associated with this slip-plane may not exist. Some planes listed in Table IV are closely related to others, for example (111) and (1111) are related to (110) and (110), respectively, by bending, and this relationship may allow dislocation lines to change direction in the crystal.

The possibility of generating edge-dislocation lines in $[10 \overline{1}]$, the alternative diagonal of the plane containing the $a$ - and $c$-axes should be considered, although polymer is not formed in this direction. The most obvious plane to consider as a slip-plane is (101), but this is probably not satisfactory as a result of molecular overlap, and there are no other possible slip-

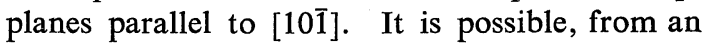
examination of the crystal structure, to visualise dislocation lines in the [011] and [110] directions

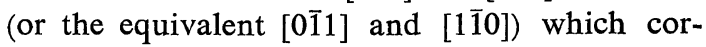
respond to continuous growth in the directions $A \leftrightarrow B, B \leftrightarrow C$ (Figure 2), i.e., with a net vector along the $b$-axis. A dislocation line parallel to [011] may be generated by inserting molecules in the plane parallel to (100), terminating along the $(11 \overline{1})$ plane, or vice versa, and parallel to [110] by inserting a half-plane of molecules in the (001) terminating along (111), or vice versa. At the present time, however, there is no evidence for polymer growth in [011] and [110].

With pentoxane the crystallographic direction corresponding to the major polymer growth direction in trioxane and tetroxane (perpendicular to the planes of the monomer molecules) is the [001] direction. Experimental studies ${ }^{12}$ have not shown any polymer growth in this direction and an examination of the crystal structure indicates that it would be difficult to produce edge-dislocation lines in this direction. In a real crystal molecules B and C (Figure 3) overlap A and $\mathrm{D}$ to such an extent as to form complete molecular layers at various levels along the $c$-axis,

Table IV. Planes associated with various slip-planes in tetroxane crystals suitable for generating edge-dislocation lines parallel to the subsidiary polymer growth directions

\begin{tabular}{lllllllllllllllllll}
\hline Growth direction & \multicolumn{1}{c}{$[001]$} & \multicolumn{9}{c}{$[100]$} & \multicolumn{4}{c}{$[101]$} \\
\hline Slip-plane & $(100)$ & $(110)$ & $(1 \overline{1} 0)$ & $(010)$ & & $(001)$ & $(011)$ & $(0 \overline{1} 1)$ & $(010)$ & & $(10 \overline{1})$ & $(\overline{1} 11)$ & $(11 \overline{1})$ & $(010)$ \\
\hline & $(010)$ & $(100)$ & $(100)$ & $(100)$ & & $(010)$ & $(001)$ & $(001)$ & $(001)$ & $(11 \overline{1})$ & $(11 \overline{1})$ & $(\overline{1} 11)$ & $(10 \overline{1})$ \\
& $(110)$ & $(1 \overline{1} 0)$ & $(110)$ & $(110)$ & & $(0 \overline{1})$ & $(0 \overline{1} 1)$ & $(011)$ & $(011)$ & $(\overline{1} 11)$ & $(010)$ & $(010)$ & \\
& $(1 \overline{1} 0)$ & & & $(1 \overline{1} 0)$ & $(011)$ & $(010)$ & $(010)$ & $(0 \overline{1} 1)$ & $(010)$ & $(10 \overline{1})$ & $(10 \overline{1})$ \\
\hline
\end{tabular}




\section{G. C. EAstmond}

preventing molecule A from 'seeing' an equivalent molecule in an adjacent unit cell in the [001] direction. There are no true slip-planes in pentoxane parallel to [001] and it is unlikely that dislocation lines running in [001] can be formed, although it is conceivable that such dislocations could be formed using combinations of the (110) and (110) planes; (110), (110) will not be true slip-planes but only suitable for pencil-glide perpendicular to the $c$-axis.

It is much easier to visualise how dislocation lines running in the main polymer growth directions, [110] and [1110], can be formed and the appropriate combinations of planes for generating dislocation lines parallel to [110] are given in Table V; in this crystal (001) is probably the major slip-plane. Subsidiary polymer growth proceeds in the equivalent [101] and [101] directions, diagonals of the plane contain-

Table V. Crystallographic planes associated with various slip-planes suitable for generating edge-dislocation lines in the [110] direction in pentoxane crystals

\begin{tabular}{ccccc}
\hline Slip plane & $(001)$ & $(1 \overline{1} 1)$ & $(1 \overline{1} 0)$ & $(\overline{1} 11)$ \\
\hline & $(1 \overline{1} 0)$ & $(\overline{1} 11)$ & $(001)$ & $(1 \overline{1} 1)$ \\
& $(\overline{1} 11)$ & $(001)$ & $(\overline{1} 11)$ & $(001)$ \\
& $(1 \overline{1} 1)$ & $(1 \overline{1} 0)$ & $(1 \overline{1} 1)$ & $(1 \overline{1} 0)$ \\
\hline
\end{tabular}

ing the $a$ - and $c$-axes. In the latter case suitable edge dislocations may be generated by introducing partial planes in (111) terminating in the (111) slip-plane, and possibly by introducing partial planes in (010) terminating in either the (111) or (111) slip-planes. The diagonals of the plane containing the $b$ - and $c$-axes are [011] and $[01 \overline{1}]$, a suitable slip-plane for generating dislocation lines in [011] is (111) and it may be possible to insert partial planes in (100) for this purpose; no polymer growth in these directions has been observed.

The number of different edge dislocations which can occur in a real crystal is limited and in the proceeding examination of the crystal structures we have probably identified all the major types of edge dislocation in the three monomers. While we have identified possible dislocation lines running in directions other than polymer growth directions in tetroxane and pentoxane, the crystallographic models illustrate that the dislocation lines which can be formed most readily run in the directions of known polymer growth. In no case has polymer growth in single crystals of these monomers been observed in a direction in which dislocation lines are unlikely.

Thus, it appears that a direct correlation exists between probable directions of dislocation lines and directions of polymer growth. In many crystals dislocations are preferable sites for reaction and we propose that dislocations in the cyclic-monomer crystals under consideration are specific sites for initiating polymerization. If this is the case then initially formed shortchain polymer will be orientated in the direction of the dislocation line, making use of the free volume available and enhanced molecular motion in the dislocation core. Since poly(oxymethylene) is readily crystallizable we may, therefore, anticipate the formation of crystalline polymer in the direction of the dislocation line. Continued growth of the crystalline polymer so formed would result in large scale polymerization with polymer crystals lying in certain preferred directions.

The above mechanism appears to be consistent with all reported experimental data on the solid-state polymerizations of trioxane, tetroxane and pentoxane. We have outlined above how, in single crystal studies, crystalline polymer will only grow in certain specific directions, as observed in practice, through specifically orientated nucleation. Formation of an edge dislocation with unit Burger's vector in these crystals requires the insertion or removal of more than one adjacent half-plane of molecules. Such half-planes need not be adjacent but may be separated by other planes, giving rise to partial dislocations with a series of local structures. The ability of a dislocation to support reaction will depend on the local structure and hence different dislocations may show different reactivity. We suggest that the relative amounts of main- and sub-crystal formation of polymer reflect the relative densities of various dislocations with structures suitable for reaction. It seems probable that the absence of polymer formation in the limited number of dislocations 
we have predicted which do not correspond to polymer growth directions is due either to a low density of such dislocations or unsuitability of local structure to support reaction.

In real crystals dislocation lines sometimes change direction so that while initiation may occur in a dislocation and propagation continue within that dislocation, so long as it continues in a straight line, the active end of the polymer may at some stage be faced with perfect lattice. Under conditions where polymerization occurs readily the strain produced in the perfect crystal by the addition of a monomer unit to the active end of a growing helical polymer molecule may cause distortion in the lattice, possibly by dislocation multiplication, thus allowing the continued linear growth of the polymer. This distortion is the same as that hitherto envisaged in lattice-controlled propagation. Here we consider that it is the initiation which occurs in specific directions and that propagation is not lattice-controlled. In this way we can understand the observations of Adler ${ }^{15}$ made on polycrystalline samples of monomer. If polymerization started in a specific direction in a small crystal then polymer growth could continue linearly across intercrystalline boundaries irrespective of the orientation of the other crystals through which the polymer grew. The resulting picture would be one of nonorientated growth, as observed by Adler. At low temperatures, where the monomer lattice is more rigid, unhindered linear growth of the polymer may not be possible.

The proposed mechanism is also consistent with kinetic observations, such as the sensitivity to impurities and irreproducibility of kinetics, since dislocations can be associated with high concentrations of impurities ${ }^{19}$ and dislocation densities are irreproducible. Proof of the ideas expressed in this paper requires a full crystallographic analysis of the monomer crystals aimed at identifying the dislocations, and their relative proportions, present in the crystals, and careful examination of partially polymerized crystals at very low extents of reaction. Canavo, et al., ${ }^{20}$ have in fact published an electron micrograph which indicates prefferential polymerization in certain regions of trioxane crystals ${ }^{20}$ and this may reflect preferred polymerization in dislocations.

Acknowledgement. The author wishes to thank the Leverhulme Trust for the award of a Visiting Fellowship in Polymer Science (Japan).

\section{REFERENCES}

1. S. Okamura, K. Hayashi, and Y. Nakamura, Isotopes and Radiation, 3, 416 (1960).

2. S. Okamura, K. Hayashi, and Y. Kitanishi, ibid., 3, 346 (1960); S. Okamura, K. Hayashi, Y. Kitanishi, and M. Nishii, ibid., 3, 510 (1960); S. Okamura, K. Hayashi, and H. Watanabe, ibid., 4, 73 (1961).

3. K. Hayashi, Y. Kitanishi, M. Nishii, and S. Okamura, Makromol. Chem., 47, 237 (1961).

4. S. Okamura, K. Hayashi, and Y. Kitanishi, $J$. Polym. Sci., 58, 925 (1962); S. Okamura and K. Hayashi, J. Chim. Phys., 59, 429 (1962); K. Hayashi, H. Ochi, and S. Okamura, J. Polym. Sci., Part A, 2, 2929 (1964).

5. G. C. Eastmond, in "Progress in Polymer Science," Vol. 2, A. D. Jenkins, Ed., Pergamon Press, Oxford, 1970; C. H. Bamford and G.C. Eastmond, Quart. Rev., 23, 271 (1969).

6. B. Arnold and G. C. Eastmond, Trans. Faraday Soc., 67, 772 (1971).

7. K. Hayashi, A. Ito, and S. Okamura, in "Large Radiation Sources for Industrial Processes," International Atomic Energy Agency, Vienna, 1969.

8. K. Hayashi and S. Okamura, U. S. Atomic Energy Commission, TID 7643, 1962, p 150; A. Komaki and T. Matsumoto, J. Polym. Sci., Part B, 1, 671 (1963).

9. K. Ueno, H. Tsukamoto, K. Hayashi, and S. Okamura, J. Polym. Sci., Part B, 5, 395 (1967).

10. G. Carrazolo, S. Leghissa, and M. Mammi, Makromol. Chem., 60, 171 (1963).

11. Y. Chatani, T. Uchida, H. Tadokoro, K. Hayashi, M. Nishii, and S. Okamura, J. Macromol. Sci., Part B, 2, 567 (1968).

12. Y. Chatani, K. Kitahama, H. Tadokoro, T. Yamauchi, and Y. Miyake, ibid., Part B, 4, 61 (1970).

13. M. Nishii, K. Hayashi, and S. Okamura, Ann. Rep. Japanese Assoc. for Radiation Research on Polymers, 7, 159 (1966); AEC-tr-6908, TID4500, U. S. Atomic Energy Commission, p 173.

14. M. Nishii, K. Hayashi, and S. Okamura, Ann. Rep. Japanese Assoc. for Radiation Research on 


\section{G. C. EASTMOND}

Polymers, 8, 151 (1967), AEC-tr-6937, TID-4500, U.S. Atomic Energy Commission, p 158.

15. G. Adler, J. Polym. Sci., Part A-1, 4, 2883 (1966).

16. D.C. Bassett, Nature, 215, 731 (1967).

17. G.C.Eastmond, E. Haigh, and B. Taylor, Trans. Faraday Soc., 65, 2497 (1969).

18. G. C. Eastmond, Molecular Crystals and Liquid
Crystals, 9, 383 (1969).

19. A.H. Cottrell, "Dislocations and Plastic Flow in Crystals," Oxford University Press, Oxford, 1953.

20. C. Cannavo, J. Deschamps, K. Hayashi, and C. Sella, Compt. Rendu Acad. Sci., Paris, Part $C, 266,777$ (1968). 